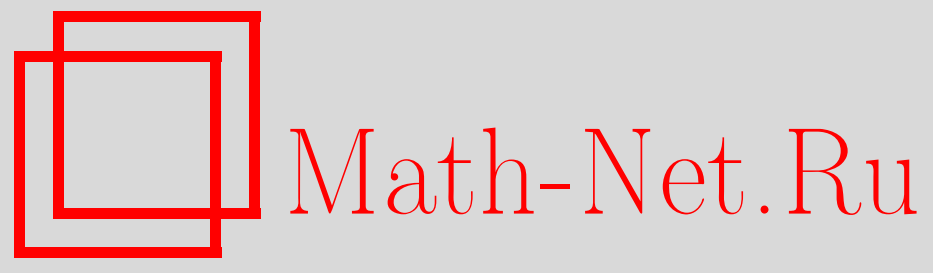

Л. В. Фомин, Описание длительной прочности растягиваемых стержней прямоугольного и круглого поперечных сечений в высокотемпературной воздушной среде, Вестн. Сам. гос. техн. ун-та. Сер. Физ.-мат. науки, 2013, выпуск 3(), 87-97

DOI: https://doi.org/10.14498/vsgtu1228

Использование Общероссийского математического портала MathNet.Ru подразумевает, что вы прочитали и согласны с пользовательским соглашением

http://www.mathnet.ru/rus/agreement

Параметры загрузки:

IP : 44.207 .124 .84

26 апреля 2023 г., 03:49:01

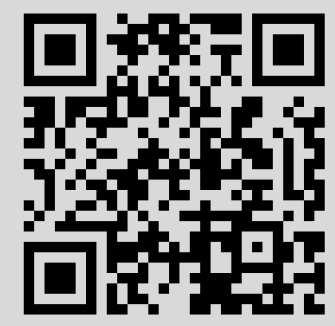


УДК 539.376; 539.434

\title{
ОПИСАНИЕ ДЛИТЕЛЬНОЙ ПРОЧНОСТИ РАСТЯГИВАЕМЫХ СТЕРЖНЕЙ ПРЯМОУГОЛЬНОГО И КРУГЛОГО ПОПЕРЕЧНЫХ СЕЧЕНИЙ В ВЫСОКОТЕМПЕРАТУРНОЙ ВОЗДУШНОЙ СРЕДЕ
}

\author{
Л. В. Фомин \\ Московский государственный университет им. М. В. Ломоносова, \\ НИИ механики МГУ, \\ 119992, Россия, Москва, Мичуринский пр-т, 1. \\ E-mail: lef1975@rambler.ru
}

\begin{abstract}
Рассматривается описание известных эксперименталъных данных по длительной прочности растягиваемых стержней прямоугольного и круглого поперечных сечений, находящихся в воздушной среде при высокой температуре. Моделирование длительной прочности растягиваемых образцов основывается на кинетической теории Ю. Н. Работнова с двумя структурными параметрамиповреждённостью и конщентрацией химических элементов окружающей средъ в стержне. Оценивается влияние поверхностного слоя, подверженного диффузии окислительной агрессивной среды, на длительную прочность образиов. Показывается необходимость учёта масштабного эфбекта, а именно размеров образиов при определении их времён до разрушения.
\end{abstract}

Ключевые слова: стержень, растяжение, длительная прочность, воздушная агрессивная среда, кинетическая теория, повреждённость, конщентрация, прямоугольное сечение, круглое сечение.

Введение. Современные материалы и конструкции авиационно-космического и энергетического машиностроения довольно часто работают в экстремальных условиях - при высоких температурах и в агрессивных средах. Поэтому к качеству и надежности конструкций, находящихся в условиях длительного высокотемпературного нагружения, предъявляются высокие требования. Всё это приводит к необходимости осуществлять прогнозирование долговечности их работы с учетом специфических факторов, одним из которых может быть агрессивная рабочая среда. Агрессивная окружающая среда оказывает существенное влияние на характеристики высокотемпературной ползучести и длительной прочности конструкционных материалов. При этом время до разрушения материалов и конструкций при воздействии этих факторов может изменяться в несколько раз.

В данной статье моделируются процессы длительной прочности материалов в агрессивной среде на основе кинетической теории Ю. Н. Работнова [1] с двумя структурными параметрами - поврежденностью $\omega$ и концентрацией $c$.

Рассматриваются известные экспериментальные данные по длительной прочности растягиваемых образцов прямоугольного поперечного сечения (опыты И. А. Одинга и З. Г. Фридмана [2]) и круглого поперечного сечения (опыты B. J. Cane, M. I. Manning, о которых сообщается в работе [3]). Указанные эксперименты проводились в высокотемпературной воздушной среде, которая в условиях, отмеченных в [2,3], является агрессивной. Оценивается

Леонид Викторович Фомин, ведущий инженер, НИИ механики МГУ. 
влияние агрессивной среды на длительную прочность растягиваемых образцов. При этом определяется зависимость времени до разрушения от средней концентрации среды в образцах. При анализе экспериментальных данных рассматриваются различные варианты аппроксимаций материальных функций.

1. Моделирование влияния агрессивной среды на длительную прочность образцов при одноосном растяжении. В данном пункте исследуется влияние агрессивной среды на длительную прочность образцов при одноосном растяжении на основе кинетической теории ползучести [1]. Рассматривается длинный образец, окруженный агрессивной средой. В [4,5] приведено решение уравнения диффузии для случая постоянного уровня агрессивной среды на поверхности стержня, основанное на введении движущегося диффузионного фронта. Далее под $c_{m}$ понимается интегральное среднее значение концентрации агрессивной среды в стержне, отнесённое к значению концентрации среды на поверхности стержня. Следуя работам [3,4], введём в кинетическое уравнение ползучести материальную функцию $f\left(c_{m}(t)\right)$. Принимая дробностепенную модель длительной прочности [6], запишем кинетическое уравнение в виде

$$
\frac{d \omega}{d t}=L\left(\frac{\sigma}{\sigma_{b}-\sigma}\right)^{n} \frac{1}{(1-\omega)^{n}} f\left(c_{m}(t)\right),
$$

где $\sigma$ - номинальное растягивающее напряжение; $\omega-$ повреждённость; $t-$ время; $\sigma_{b}$ - предел кратковременной прочности при соответствующей температуре; $L, n$ - материальные константы.

Проинтегрируем соотношение (1):

$$
\frac{1-(1-\omega)^{n+1}}{n+1}=L\left(\frac{\sigma}{\sigma_{b}-\sigma}\right)^{n} \int_{0}^{t} f\left(c_{m}(t)\right) d t .
$$

Согласно концепции Ю. Н. Работнова, разрушение образца при ползучести наступает при достижении предельного значения повреждённости $(\omega=1)$. Используя данный критерий длительной прочности, из соотношения (2) получим

$$
1=L(n+1)\left(\frac{\sigma}{\sigma_{b}-\sigma}\right)^{n} \int_{0}^{t^{*}} f\left(c_{m}(t)\right) d t,
$$

где $t^{*}$ - время до разрушения образца при ползучести с учётом воздействия агрессивной среды.

Пусть $c_{m}(t) \equiv 0$ (окружающая агрессивная среда отсутствует). При этом $f\left(c_{m}(t)\right)$ должна быть задана таким образом, чтобы соответствовать условию $f\left(c_{m}(t)\right) \equiv 1$.

Используя перечисленные условия в соотношении (3), получим

$$
t_{\text {без среды }}^{*}=\left[L(n+1)\left(\frac{\sigma}{\sigma_{b}-\sigma}\right)^{n}\right]^{-1} \text {, }
$$

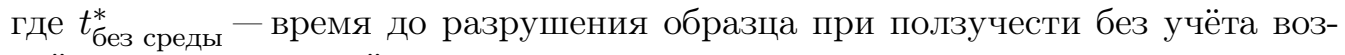
действия агрессивной среды. 
Возвращаясь к соотношению (3), получим искомую взаимосвязь времен до разрушения образца при ползучести с учетом влияния агрессивной среды $t^{*}$ и без его учета $t_{\text {без среды: }}^{*}$

$$
t_{\text {без среды }}^{*}=\int_{0}^{t^{*}} f\left(c_{m}(t)\right) d t .
$$

\section{2. Описание длительной прочности образцов прямоугольного поперечного} сечения (опыты И. А. Одинга и З. Г. Фридмана). В качестве примера моделирования влияния агрессивной окружающей среды на характеристики длительной прочности металлов рассмотрим результаты опытов, проведённых И. А. Одингом и З. Г. Фридманом в воздушной среде [2]. В указанных экспериментах использовались плоские образцы из отожжённой мягкой углеродистой стали Ст. 3. Рабочая длина каждого образца составляла 28 мм, ширина - 6 мм. Толщина образцов $H$ принимала различные значения от 0,15 мм до 2 мм, т.е. изменялась более чем в 13 раз. Испытания на ползучесть вплоть до разрушения проводились при температуре $T=450{ }^{\circ} \mathrm{C}$ и номинальных напряжениях $\sigma=220 \div 270$ МПа, колебания температуры не превышали $\pm 5^{\circ} \mathrm{C}$. Анализ результатов испытаний показывает, что изменение $H$ от 2,0 до 1,0 мм практически не влияет на характеристики ползучести и длительной прочности образцов, так что можно считать, что при толщине образцов $H=1$ мм и более влияние окружающей среды на длительную прочность несущественно. Экспериментальные времена до разрушения $t_{\ni}^{*}$ для различных толщин представлены в табл. 1. Уменьшение толщины $H$ от 1,0 до 0,15 мм при любых напряжениях приводит к уменьшению $t_{\ni}^{*}$ в 6-8 раз.

Таблица 1

Результаты опытов по длительной прочности И. А. Одинга и З. Г. Фридмана [2]

\begin{tabular}{|c|c|c|c|c|c|c|c|}
\hline \multicolumn{2}{|c|}{$\sigma=220 \mathrm{MПа}$} & \multicolumn{2}{|c|}{$\sigma=235 \mathrm{MПа}$} & \multicolumn{2}{|c|}{$\sigma=250 \mathrm{MПа}$} & \multicolumn{2}{|c|}{$\sigma=270 \mathrm{MПа}$} \\
\hline$H$, мм & $t_{\ni}^{*}$, час & $H$, мм & $t_{\ni}^{*}$, час & $H$, мм & $t_{\ni}^{*}$, час & $H$, мм & $t_{\ni}^{*}$, час \\
\hline$H_{1}=0,15$ & 98 & 0,15 & 60 & 0,15 & 26 & 0,15 & 12 \\
\hline$H_{2}=0,2$ & - & 0,2 & 54 & 0,2 & 32 & 0,2 & 16 \\
\hline$H_{3}=0,3$ & 140 & 0,3 & 63 & 0,3 & 42 & 0,3 & 23 \\
\hline$H_{4}=0,5$ & 679 & 0,5 & 234 & 0,5 & 115 & 0,5 & 40 \\
\hline$H_{5}=1,0$ & 800 & 1,0 & 350 & 1,0 & 170 & 1,0 & 77 \\
\hline \multicolumn{2}{|c|}{$t_{0}^{*}=800$, час } & \multicolumn{2}{|c|}{$t_{0}^{*}=350$, час } & \multicolumn{2}{|c|}{$t_{0}^{*}=170$, час } & \multicolumn{2}{|c|}{$t_{0}^{*}=77$, час } \\
\hline
\end{tabular}

Авторы [2] обращают внимание на различную роль поверхностных и внутренних слоёв при ползучести. Они отмечают, что первые трещины при длительном разрушении металла в условиях ползучести появляются, как правило, на поверхности образца. Это свидетельствует о том, что процессы разрушения поверхностных слоев образца протекают по своей интенсивности с опережением по сравнению с внутренними слоями. Масштабный эффект заметно усиливается при уменьшении толщины образцов, при этом возрастает относительная объёмная доля их поверхностного слоя.

По мнению автора настоящей статьи, причиной разрушения поверхностных слоев является тот факт, что при указанной температуре поверхностные слои ослаблены агрессивным воздействием кислорода, содержащимся в воздушной среде, который образует окислы на поверхности. 
Для анализа экспериментальных данных зададим три различных варианта функции $f\left(c_{m}(t)\right)$ в виде линейной, экспоненциальной и степенной (квадратный корень) функций:

$$
\begin{gathered}
f\left(c_{m}(t)\right)=\exp \left(a_{1} c_{m}(t)\right), \\
f\left(c_{m}(t)\right)=1+a_{2} c_{m}(t), \\
f\left(c_{m}(t)\right)=1+a_{3} \sqrt{c_{m}(t)} .
\end{gathered}
$$

В $[4,5]$ предложено приближенное решение уравнения диффузии, основанное на введении диффузионного фронта. Интегральное среднее значение безразмерной концентрации $c_{m}(t)$ стержня прямоугольного поперечного сечения в виде тонкой полосы толщиной $H$ имеет следующий вид $[4,5]$ :

$$
c_{m}(t)=\left\{\begin{array}{cll}
\frac{1}{3} \sqrt{\frac{48 D}{H^{2}} t}, & \text { если } & 0<t \leqslant \frac{H^{2}}{48 D} \\
1-\frac{2}{3} \exp \left(\frac{1}{4}\left(1-\frac{48 D}{H^{2}} t\right)\right), & \text { если } t>\frac{H^{2}}{48 D}
\end{array}\right.
$$

где $D=$ const - коэффициент диффузии.

Определим материальные константы $L$ и $n$, входящие в соотношение (1). Эти константы определяются из соотношения (4) на основе обработки значений экспериментальных времен до разрушения $t_{\ni}^{*}$ при $H=1$ мм, для которых характеристики длительной прочности практически не зависят от окружающей среды. С помощью метода наименьших квадратов получены следующие значения материальных констант: $L=1,04 \cdot 10^{-4}, n=4,39$.

Для поиска оптимального варианта функции $f\left(c_{m}(t)\right)$ и определения соответствующих констант $a_{1}, a_{2}, a_{3}$, входящих в соотношения $(1),(6)-(8)$, были рассчитаны теоретические значения времен до разрушения $t^{*}$ для каждого вида предложенных аппроксимаций $f\left(c_{m}(t)\right)$ с учётом минимального суммарного отклонения $S$ полученных теоретических времен разрушения $t^{*}$ от экспериментальных $t_{\ni}^{*}$ по формуле

$$
S=\frac{1}{N} \sum_{i=1}^{N}\left(\frac{t^{*}-t_{\ni}^{*}}{t^{*}+t_{\ni}^{*}}\right)_{i}^{2}
$$

где $N=11$ - суммарное количество экспериментальных точек для опытов с толщинами полос $H=0,15 \div 0,3 \mathrm{~mm}, t^{*}$ - теоретическое время до разрушения.

Необходимо отметить, что при анализе экспериментальных данных были исключены значения $t_{\ni}^{*}$ при $=0,5$ мм с незначительным масштабным эффектом.

Теоретическое время до разрушения $t^{*}$ определялось в соответствии с формулой (5), в которой время до разрушения образцов без агрессивной среды определялось на основе соотношения (4) с использованием найденных констант $L=1,04 \cdot 10^{-4}$ и $n=4,39$ для полосы толщиной $H=1$ мм.

Результаты расчета с использованием трех видов аппроксимаций (6)-(8) приведены в табл. 2. 


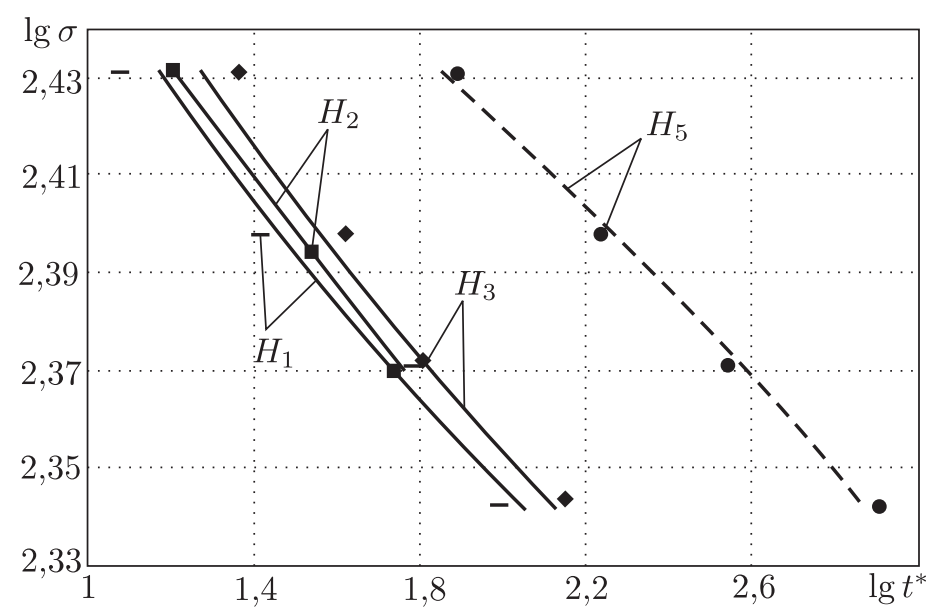

Диаграммы длительной прочности: точки - эксперимент; сплошные линии - расчётные кривые с учётом степенной зависимости; штриховая линия - аппроксимация с помощью метода наименьших квадратов для толщины $H_{5}=1$ мм без учёта агрессивной среды

Таблица 2

В соответствии с табл. 2, минимальное значение суммарного отклонения $S$ определяется для степенной аппроксимации $f\left(c_{m}(t)\right)$ вида (8).

На рисунке приведены графики длительной прочности, построенные в соответствии с данными табл. 2, для степенной зависимости $f\left(c_{m}(t)\right)$.

В случае точного решения уравнения диффузии [4,5] интегральное

Значения материальных констант и суммарное отклонение теоретических времен до разрушения от экспериментальных

\begin{tabular}{c|c|c}
\hline \multicolumn{2}{c}{ Значение материальных констант } \\
\hline$a_{1}$ & $a_{2}$ & $a_{3}$ \\
\hline 3,10 & 9,50 & 6,72 \\
\hline
\end{tabular}

Суммарное отклонение $S$

\begin{tabular}{c|c|c}
\hline $\exp \left(a_{1} c_{m}(t)\right)$ & $1+a_{2} c_{m}(t)$ & $1+a_{3} \sqrt{c_{m}(t)}$ \\
\hline 0,018 & 0,0042 & 0,0036 \\
\hline
\end{tabular}
среднее значение безразмерной концентрации $c_{m}(t)$ для стержня в виде тонкой полосы прямоугольного поперечного сечения толщиной $H$ имеет следующий вид:

$$
\bar{c}_{m}(t)=1-\frac{8}{\pi^{2}} \sum_{k=0}^{\infty} \frac{1}{(2 k+1)^{2}} \exp \left(-\frac{(2 k+1)^{2} \pi^{2}}{48} \cdot \frac{48 D}{H^{2}} t\right) .
$$

В соответствии с точным решением (11) был произведён аналогичный расчёт материальных констант и суммарного отклонение теоретических времен до разрушения от экспериментальных. Результаты расчёта представлены в табл. 3.

В соответствии с расчётными данными, представленными в табл. 2 и 3, сравнение результатов по приближенному (9) и точному (11) решениям уравнения диффузии показало близкие результаты.

Покажем, что учёт масштабного эффекта существенно влияет на величину суммарного отклонения $S$ теоретических времён до разрушения $t^{*}$ от экспериментальных $t_{\ni}^{*}$ и, как следствие, приводит к более корректному описанию длительной прочности стержней. Для того чтобы это показать, рассмотрим полученные И. А. Одингом и З. Г. Фридманом экспериментальные 
значения времен до разрушения $t_{\ni}^{*}$ вне зависимости от толщин образцов, а зависящими только от напряжения $\sigma$.

Таблица 3

Значение материальных констант и суммарное отклонение теоретических времен до разрушения от экспериментальных на основе точного решения уравнения диффузии

\begin{tabular}{c|c|c}
\hline \multicolumn{3}{c}{ Значение материальных констант } \\
\hline$a_{1}$ & $a_{2}$ & $a_{3}$ \\
\hline 3,215 & 9,93 & 6,915 \\
\hline \multicolumn{3}{c}{ Суммарное отклонение $S$} \\
\hline $\exp \left(a_{1} c_{m}(t)\right)$ & $1+a_{2} c_{m}(t)$ & $1+a_{3} \sqrt{c_{m}(t)}$ \\
\hline 0,018 & 0,004098 & 0,00373 \\
\hline
\end{tabular}

Применим аппроксимацию вида (4) к описанию всей совокупности экспериментальных точек. Для этого определим значения материальных констант, входящих в соотношение (4), и далее на основе полученных значений теоретических времен до разрушения $t^{*}$ подсчитаем величину суммарного отклонения $S$ по формуле (10).

Для реализации предложенного подхода используем следующую методику. Согласно справочнику [7] примем предел прочности Ст. 3 при $T=450{ }^{\circ} \mathrm{C}$ равным $\sigma_{b}=400$ МПа. Предварительно с помощью метода наименьших квадратов найдём материальные константы $L_{i}$ и $n_{i}$ аппроксимации (4) для разных значений толщин образцов $\left(H_{i}=0,15 \div 0,3\right)$ на трёх образцах. Затем на основе среднего значения $n$ определим константу $L$ при помощи минимизации значения суммарного отклонения $S$, подсчитанного для всей совокупности экспериментальных точек $t_{\ni}^{*}$ без учёта их зависимости от толщины образцов.

В результате вычислений по данной методике были получены следующие значения: $n=3,53, L=7,5 \cdot 10^{-4}, S=0,011$.

Сравнение суммарного отклонения $S$ теоретических времен до разрушения $t^{*}$ с экспериментальными $t_{\ni}^{*}$ показало преимущество учёта масштабного эффекта при описании длительной прочности. Об этом свидетельствует тот факт, что отклонение $S$ при учёте масштабного эффекта в 3 раза меньше, чем без его учёта.

3. Описание длительной прочности образцов круглого поперечного сечения (опыты В. J. Cane, M. I. Manning). В данном пункте рассматриваются ползучесть и длительная прочность при растяжении цилиндрических образцов под действием высокой температуры в воздушной среде. Как было отмечено ранее, воздушная среда при высокой температуре является агрессивной.

Рассмотрим процесс диффузии агрессивной среды при растяжении цилиндрического образца на основе введения диффузионного фронта среды [3, 4]. Введём безразмерные переменные

$$
\bar{r}=r / R, \quad \bar{c}=c / c_{0}, \quad \tilde{t}=D t / R^{2},
$$

где $r$-расстояние от оси симметрии образца до произвольной точки, $R-$ радиус поперечного сечения образца, $D$ - коэффициент диффузии, $t$ - время. В безразмерных переменных (12) уравнение диффузии и краевые условия для него принимают следующий вид:

$$
\frac{\partial \bar{c}(\bar{r}, \tilde{t})}{\partial \tilde{t}}=\frac{1}{\bar{r}} \frac{\partial}{\partial \bar{r}}\left(\bar{r} \frac{\partial \bar{c}}{\partial \bar{r}}\right), \quad \bar{c}(\bar{r}, 0)=0, \quad \bar{c}(1, \tilde{t})=1, \quad \frac{\partial \bar{c}}{\partial \tilde{t}}(0, \tilde{t})=0 .
$$


Будем искать приближённое решение уравнения (13) с помощью введения диффузионного фронта среды. Введём координату фронта $\bar{l}(\tilde{t})$ так, чтобы $\bar{l}(0)=1$ при $r=R$ (поверхность образца) и $\bar{l}\left(\tilde{t}_{0}\right)=0$ при $r=0$ (центр симметрии образца). Здесь $\tilde{t}_{0}$ - безразмерное время достижения диффузионным фронтом центра симметрии образца, т.е. заполнения диффузионной средой всего поперечного сечения.

Рассмотрим две последовательные стадии решения: первая стадия - до момента заполнения фронтом всей области поперечного сечения образца $\left(0<\tilde{t} \leqslant \tilde{t}_{0}\right) ;$ вторая - стадия насыщения $\left(\tilde{t}>\tilde{t}_{0}\right)$.

Зависимости безразмерной концентрации $\bar{c}$ от безразмерных координаты $\bar{r}$ при любом времени $\tilde{t}$ зададим в виде параболических зависимостей, которые удовлетворяют граничным условиям (13):

$$
\bar{c}(\bar{r}, \tilde{t})=\left\{\begin{array}{ccl}
0, & \text { если } & 0 \leqslant \bar{r} \leqslant \bar{l}(\tilde{t}) \\
(\bar{r}-\bar{l}(\tilde{t}))^{2} /(1-\bar{l}(\tilde{t}))^{2}, & \text { если } & \bar{l}(\tilde{t}) \leqslant \bar{r} \leqslant 1
\end{array}\right.
$$

при $0<\tilde{t} \leqslant \tilde{t}_{0}($ первый этап) и

$$
\bar{c}(\bar{r}, \tilde{t})=B(\tilde{t})+(1-B(\tilde{t})) \bar{r}^{2}, \quad \text { если } \quad 0 \leqslant \bar{r} \leqslant 1
$$

при $\tilde{t}>\tilde{t}_{0}$ (второй этап).

Определим функции $\bar{l}(\tilde{t})$ и $B(\tilde{t})=\bar{c}(0, \tilde{t})$ из интегрального уравнения диффузии:

$$
\int_{0}^{1}\left(\frac{\partial \bar{c}}{\partial \tilde{t}}-\frac{1}{\bar{r}} \frac{\partial}{\partial \bar{r}}\left(\bar{r} \frac{\partial \bar{c}}{\partial \bar{r}}\right)\right) \bar{r} d \bar{r}=0
$$

Подставляя (14) и (15) в (16), определим функции $\bar{l}(\tilde{t})$ и $B(\tilde{t})$, а затемконцентрацию $\bar{c}(\bar{r}, \tilde{t})$. Уравнения $(14)-(16)$ приводят к следующим зависимостям координаты диффузионного фронта $\bar{l}(\tilde{t})$ и концентрации $B(\tilde{t})$ от времени $\tilde{t}$ :

$$
\left\{\begin{array}{l}
\frac{d \bar{l}}{d \tilde{t}}=-\left.\frac{k(k+1)(k+2)}{(1-\bar{l})(k+2 \bar{l})}\right|_{k=2}=-\frac{12}{(1-\bar{l})(1+\bar{l})}, \quad 0<\tilde{t} \leqslant \tilde{t}_{0} \\
\tilde{t}=\left.\frac{(1-\bar{l})^{2}(2+3 k+4 \bar{l})}{6 k(k+1)(k+2)}\right|_{k=2}=\frac{(1-\bar{l})^{2}(2+\bar{l})}{36}, \quad 0<\tilde{t} \leqslant \tilde{t}_{0} \\
B(\tilde{t})=1-\left.\exp \left(-2(k+2)\left(\tilde{t}-\tilde{t}_{0}\right)\right)\right|_{k=2}=1-\exp \left(-8\left(\tilde{t}-\tilde{t}_{0}\right)\right), \quad \tilde{t}>\tilde{t}_{0} \\
\tilde{t}_{0}=\left.\tilde{t}(\bar{l}=0) \frac{(1-\bar{l})^{2}(2+3 k+4 \bar{l})}{6 k(k+1)(k+2)}\right|_{k=2}=\frac{1}{18}
\end{array}\right.
$$

Вычислим зависимость интегрального среднего уровня концентрации в поперечном сечении образца

$$
\bar{c}_{m}(\tilde{t})=2 \int_{0}^{1} \bar{c}(\bar{r}, \tilde{t}) \bar{r} d \bar{r}
$$

с помощью (14), (15), (17) и (18) в безразмерном времени:

$$
\bar{c}_{m}(\tilde{t})=\left\{\begin{array}{cll}
(1-\bar{l})(3+\bar{l}) / 6, & \text { если } & 0 \leqslant \tilde{t} \leqslant \tilde{t}_{0} \\
1-\frac{1}{2} \exp \left(8\left(\tilde{t}_{0}-\tilde{t}\right)\right), & \text { если } & \tilde{t}>\tilde{t}_{0} .
\end{array}\right.
$$


С учётом введённого соотношения для безразмерного времени (12) последнее соотношение можно представить в виде

$$
\bar{c}_{m}(t)=\left\{\begin{array}{cll}
(1-\bar{l})(3+\bar{l}) / 6, & \text { если } & 0 \leqslant t \leqslant \tilde{t}_{0} R^{2} / D \\
1-\frac{1}{2} \exp \left(8\left(\tilde{t}_{0}-t D / R^{2}\right)\right), & \text { если } & t>\tilde{t}_{0} R^{2} / D .
\end{array}\right.
$$

Так как коэффициент $D$ очень мал, в уравнении (19) можно рассматривать только первую стадию. При этом $\tilde{t}=t D / R^{2} \ll 1$ и второе соотношение (17) можно оценить так:

$$
\bar{t}=(1-\bar{l})^{2}(2+\bar{l}) / 36 \ll 1
$$

т. е. можно считать, что $1-\bar{l} \ll 1$. Положим $\bar{l}=1-\delta$, где $\delta \ll 1$, и преобразуем второе соотношение в (17) к виду

$$
-\delta^{3}+3 \delta^{2}=36 \tilde{t}
$$

из которого, пренебрегая величиной $\delta^{3}$, принимаем $\delta \approx \sqrt{12 \tilde{t}}$. Тогда

$$
\bar{l}=1-\delta=1-\sqrt{12 \tilde{t}}
$$

Выразим в первом уравнении $(19) \bar{c}_{m}(t)$ через $\delta$ :

$$
\bar{c}_{m}(t)=\frac{(1-\bar{l})(3+\bar{l})}{6}=\frac{\delta(4-\delta)}{6} \approx \frac{2}{3} \delta=\frac{4}{\sqrt{3}} \sqrt{\frac{D}{R^{2}} t} .
$$

Рассмотрим опыты В. J. Cane, M. I. Manning по ползучести и длительной прочности при растяжении цилиндрических образцов при одинаковом напряжении, о которых сообщается в работе [3], применительно к нашей модели.

\section{Таблица 4}

Экспериментальные времена до разрушения

\begin{tabular}{c|c}
\hline Радиус $R$, мм & Время до разрушения $t_{\ni}^{*}$, час \\
\hline 1,25 & 160 \\
2,5 & 241 \\
5 & 289 \\
25 & 446 \\
\hline вакуум & 496 \\
\hline
\end{tabular}
первого пункта настоящей работы. В соответствии с табл. 1 примем для расчёта время до разрушения без влияния среды $t_{\text {без среды }}^{*} 496$ час. Коэффициент диффузии $D$ примем равным $10^{-4} \mathrm{mм}^{2} /$ час.

Аналогично предыдущему пункту статьи для анализа экспериментальных данных зададим три различных варианта функций $f\left(c_{m}(t)\right)$ :

$$
\begin{gathered}
f\left(c_{m}(t)\right)=\exp \left(b_{1} c_{m}(t)\right), \\
f\left(c_{m}(t)\right)=1+b_{2} c_{m}(t),
\end{gathered}
$$




$$
f\left(c_{m}(t)\right)=1+b_{3} \sqrt{c_{m}(t)} .
$$

Для поиска оптимального варианта функции $f\left(c_{m}(t)\right)$ и определения соответствующих констант $b_{1}, b_{2}, b_{3}$, входящих в соотношения $(20)-(22)$, были рассчитаны теоретические значения времён до разрушения $t^{*}$ для каждого вида предложенных аппроксимаций $f\left(c_{m}(t)\right)$ с учётом минимального суммарного отклонения $S$ полученных теоретических времен до разрушения $t^{*}$ от экспериментальных $t_{\ni}^{*}$ :

$$
S=\frac{1}{N} \sum_{i=1}^{N}\left(\frac{t^{*}-t_{\ni}^{*}}{t^{*}+t_{\ni}^{*}}\right)_{i}^{2}
$$

где $N=4$ - суммарное количество экспериментальных точек, $t^{*}$ - теоретическое время до разрушения, $t_{\ni}^{*}$ - экспериментальное время до разрушения образца.

Результаты расчёта с использованием трёх видов аппроксимаций (20)-(22) приведены в табл. 5.

В соответствии с табл. 5 минимальное значение суммарного отклонения $S$ определяется для линейного вида (21) аппроксимации $f\left(c_{m}(t)\right)$. В табл. 6 представлены экспериментальные и расчётные теоретические значения времен до разрушения в соответствии с линейной аппроксимацией вида (21).

Таблица 5

Значения материальных констант и суммарное отклонение теоретических времен до разрушения от экспериментальных

\begin{tabular}{c|c|c}
\hline \multicolumn{3}{c}{ Значение материальных констант } \\
\hline$b_{1}$ & $b_{2}$ & $b_{3}$ \\
\hline 7,33 & 12,55 & 3,78 \\
\hline
\end{tabular}

Суммарное отклонение $S$

\begin{tabular}{c|c|c}
\hline $\exp \left(b_{1} c_{m}(t)\right)$ & $1+b_{2} c_{m}(t)$ & $1+b_{3} \sqrt{c_{m}(t)}$ \\
\hline $1,11 \cdot 10^{-3}$ & $4,16 \cdot 10^{-4}$ & $6,11 \cdot 10^{-3}$ \\
\hline
\end{tabular}

Таблица 6

Экспериментальные и теоретические времена до разрушения цилиндрических образцов

\begin{tabular}{c|c|c}
\hline Радиус $R$, мм & $\begin{array}{c}\text { Экспериментальное время } \\
\text { до разрушения } t_{\Im}^{*}, \text { час }\end{array}$ & $\begin{array}{c}\text { Теоретическое время } \\
\text { до разрушения } t^{*}, \text { час }\end{array}$ \\
\hline 1,25 & 160 & 165,8 \\
\hline 2,5 & 241 & 228,7 \\
\hline 5 & 289 & 297,6 \\
\hline 25 & 446 & 427,6 \\
\hline
\end{tabular}

Таким образом, как показано в табл. 6, экспериментальные и теоретические времена до разрушения увеличиваются с ростом радиусов цилиндрических образцов. По мнению автора настоящей статьи, этот масштабный эффект связан с различной относительной долей проникновения высокотемпературной агрессивной воздушной среды в материал цилиндрических образцОВ.

Обсуждение результатов и выводы. Определены характеристики длительной прочности стержней в условиях растяжения, находящихся в агрессивной высокотемпературной воздушной окружающей среде. Исследования проведены на основе кинетической теории Ю. Н. Работнова с двумя структурными параметрами: повреждённостью и концентрацией диффундирующих элементов окружающей среды в материале стержней. Учёт влияния агрессивной 
среды проводится путём введения в кинетическое уравнение интегральной средней концентрации элементов среды в материале образцов. Интегральная средняя концентрация определяется на основе введения диффузионного фронта среды.

С помощью минимизации суммарного отклонения теоретических времен до разрушения от экспериментальных определен оптимальный вид материальной функции и её параметров в зависимости от геометрии поперечного сечения образцов: в случае образцов прямоугольного поперечного сечения степенной вид функции, а в случае образцов круглого поперечного сечения линейный вид.

Проведено сравнение материальных параметров в случае точного и приближённого решения уравнения диффузии. Получены близкие результаты.

На основе учёта масштабного эффекта показана зависимость времен до разрушения цилиндрических образцов от средней концентрации агрессивной среды в материале образцов.

На примере описания длительной прочности плоских образцов прямоугольного поперечного сечения показано сравнение суммарного отклонения теоретических времён до разрушения $t^{*}$ с экспериментальными $t_{\ni}^{*}$. Это сравнение показало справедливость учёта масштабного эффекта при описании длительной прочности. Об этом свидетельствует тот факт, что отклонение $S$ при учёте масштабного эффекта в 3 раза меньшее, чем без учёта. Масштабный эффект заметно усиливается при уменьшении толщины образцов по причине увеличения относительной объемной доли поверхностного слоя, подверженного влиянию агрессивной среды.

Проведённый анализ показал, что учёт влияния агрессивной среды на элементы конструкций при ползучести приводит к наблюдаемому в эксперименте уменьшению времени до разрушения в условиях действия растягивающих нагрузок.

Автор выражает благодарность профессору А. М. Локощенко за постановку задачи и полезные советы. Работа выполнена при финансовой поддержке РФФИ (проекты №11-0800007-а и 11-08-01015-а).

\section{БИБЛИОГРАФИЧЕСКИЙ СПИСОК}

1. Ю. Н. Работнов, Ползучесть элементов конструкции. М.: Наука, 1966. 752 с. [Yu. N. Rabotnov, Creep of Structural Elements. Moscow: Nauka, 1966. 752 pp.]

2. И. А. Одинг, 3. Г. Фридман, "Роль поверхностных слоев при длительном разрушении металлов в условиях ползучести" // Завод. лаб., 1959. Т. 25, № 3. С. 329-332. [I. A. Oding, Z. G. Fridman, "Role of surface layers in long-term fracture of metals under creep conditions" // Zavod. Lab., 1959. Vol. 25, no. 3. Pp. 329-332].

3. B. J. Cane, R. D. Townsend, "Prediction of Remaining Life in Low-Allow Steels" / In: Flow and Fracture at Elevated Temperatures; ed. R. Raj. Ohio: Amer. Soc. For Metals, 1985. Pp. 279-316.

4. А. М. Локощенко, Ползучесть и длительная прочность металлов в агрессивных средах. М.: МГУ, 2000. 178 с. [A. M. Lokoshchenko, Creep and Long-Term Strength of Metals in Corrosive Media. Moscow: Moscow State Uni., 2000. 178 pp.]

5. А. М. Локощенко, Моделирование ползучести и длительной прочности металлов. М.: МГИУ, 2007. 264 с. [A. М. Lokoshchenko, Modeling of Creep and Long-Term Strength of Metals. Moscow: Moscow State Industr. Uni., 2007. 264 pp.]

6. С. А. Шестериков, М. А. Юмашева, "Конкретизация уравнения состояния в теории ползучести" // Изв. Акад. наук СССР. Сер. Механика твердого тела, 1984. № 1. С. 86- 
92. [S. A. Shesterikov, M. A. Yumasheva, "Concretization of the equation of state in the theory of creep" // Izv. Akad. Nauk SSSR, Seriya Mekhanika Tverdogo Tela, 1984. no. 1. Pp. 96-101].

7. В. Г. Сорокин, М. А. Гервасвев, В. С. Паеев и др., Стали и сплавы. Марочник: Справ. изд. / ред. В. Г. Сорокин, М. А. Гервасьев. М.: Интермет инжениринг, 2001. 608 с. [V. G. Sorokin, M. A. Gervasiev, V. S. Paeev et al., Steels and alloys. Grade List. Moscow: Intermet Engineering, 2001. 608 pp.]

Поступила в редакцию 29/V/2013;

в окончательном варианте - 27/VIII/2013.

MSC: 74A45; 74D10, 74K10

\title{
DESCRIPTION OF CREEP RUPTURE STRENGTH OF TENSILE ROD WITH RECTANGULAR AND CIRCULAR CROSS-SECTION AT HIGH TEMPERATURE AIR MEDIA
}

\author{
L. V. Fomin \\ M. V. Lomonosov Moscow State University, \\ Research Institute of Mechanics, \\ 1, Michurinsky pr., Moscow, 119192, Russia. \\ E-mail: lef1975@rambler.ru
}

Description of known experimental data on the creep rupture strength of tensile rectangular and circular cross-sections rods at high temperature in air media is considered. Simulation of the creep rupture strength of tensile specimens is based on the $Y$ u. N. Rabotnov's kinetic theory with two structural parameters - damage and concentration of the chemical elements of the medium in the rod's material. It has been shown on the basis of experimental data that the scale effect and in particular the relative volume fraction of the surface layer of the rod exposed to the aggressive medium influence on the characteristics of creep rupture strength.

Key words: rod, tension, creep rupture strength, air aggressive media, kinetic theory, damage, concentration, rectangular cross-section, circular cross-section.

Original article submitted $29 / \mathrm{V} / 2013$ revision submitted $27 / \mathrm{VIII} / 2013$.

Leonid V. Fomin, Leading Engineer, Research Institute of Mechanics. 